\title{
AISLAMIENTO E IDENTIFICACIÓN DE HONGOS NEMATÓFAGOS NATIVOS DE ZONAS ARROCERAS DE COSTA RICA ${ }^{1}$
}

\author{
Walter Peraza-Padilla², Martha Orozco-Aveces ${ }^{2}$,Alejandro Esquivel-Hernández ${ }^{2}$, German Rivera-Coto², \\ Fabio Chaverri-Fonseca ${ }^{2}$
}

\section{RESUMEN}

Aislamiento e identificación de hongos nematófagos nativos de zonas arroceras de Costa Rica. El objetivo de la presente investigación fue identificar hongos nematófagos en fincas arroceras de Costa Rica. El estudio se realizó entre los meses de abril del 2008 a agosto de 2009. Fueron analizadas muestras de suelo provenientes de tres regiones productoras de arroz de Costa Rica: Pacífico Central, Huetar Atlántica y Chorotega, mediante el método de espolvoreado en placa en búsqueda de hongos nematófagos. Los hongos aislados fueron identificados como Trichoderma sp., Penicillium sp., Paecilomyces sp., Fusarium oxysporum, Fusarium tabacinum, Fusarium sp., Aspergillus sp., Monacrosporium sp., Gonatobotrys sp., Geotrichium sp., Phythium sp., Cladosporium sp., Curvularia sp., Phoma sp., Oomycete y micelio estéril. Los resultados sugieren una alta diversidad de hongos nematófagos con potencial para ser utilizados como agentes de control biológico.

Palabras clave: Nematodos fitoparásitos, Trichoderma sp., Monacrosporium sp., Paecilomyces sp., Fusarium oxysporum.

\begin{abstract}
Isolation and identification of nematophagous fungi from native rice-producing areas of Costa Rica. The objective of this study was to isolate and identify nematophagous fungi from rice-producing areas of Costa Rica. The study was conducted between April 2008 to August 2009. Soil from three rice-producing regions of Costa Rica, Central Pacific, Atlantic and Region Huetar Chorotega were analyzed by the sprinkling plate method in search of nematophagous fungi. The fungal isolated were identified as Trichoderma sp., Paecilomyces sp., Fusarium oxysporum, Fusarium tabacinum, Fusarium sp., Monacrosporium sp., Aspergillus sp., Geotrichum sp., Phythium sp., Cladosporium, Curvularia sp., Phoma sp., Oomycete and sterile mycelium. The results suggest a high diversity of nematophagous fungi with potential for use as biological control agents.
\end{abstract}

Key words: Nematophagous fungi, Trichoderma sp., Monacrosporium sp., Paecilomyces sp., Fusarium oxysporum.

\section{INTRODUCCIÓN}

El arroz (Oryza sativa L.) es el principal cereal cultivado en Costa Rica con una superficie aproximada de
63 mil hectáreas (MAG 2011). A pesar del incremento en los rendimientos con el uso de mejoras genéticas, existe una incidencia de plagas que se ve reflejada directamente en la calidad y la cantidad de las cosechas.

\footnotetext{
1 Recibido: 28 de marzo, 2011. Aceptado: 3 de octubre, 2011. Resultados parciales de la tesis de Maestría del primer autor en Agricultura Alternativa con Mención en Agricultura Ecológica.

2 Laboratorio de Nematología, Escuela de Ciencias Agrarias. wperaza@una.ac.cr, aesquive@una.ac.cr, Laboratorio de Microorganismos de Suelo y Control Biológico. marthaoa2001@yahoo.com.mx, Laboratorio de Fitopatología, Escuela de Ciencias Agrarias. griver@una.ac.cr y Escuela de Ciencias Ambientales. fchaverr@una.ac.cr Universidad Nacional. 83-3000. Heredia, Costa Rica.
} 
El control químico sigue utilizándose como la opción más rápida para enfrentar los problemas fitosanitarios. Sin embargo, la implicación ambiental es un tema muy discutido actualmente, $\mathrm{y}$ ante este panorama, el control biológico (CB) surge como una alternativa promisoria en el control de patógenos y plagas incluidos los nematodos fitoparásitos. Los antagonistas de estos nematodos han sido localizados en un amplio rango de organismos que incluyen hongos, bacterias, virus, plantas, protozoarios, turbelarios, tardígrados, ácaros e inclusive otros nematodos. Jansson y LópezLlorca (2001) afirman que de una a cinco especies de estos microrganismos son recobradas de una muestra de suelo. Dentro de este amplio grupo están los hongos nematófagos, organismos que han sido ampliamente estudiados y según Chen y Dickson (2004), los más importantes en la regulación de poblaciones de nematodos. Desde la descripción de Arthobotrys oligospora por Zopf (1988), se han realizado muchos estudios acerca de la taxonomía, ecología y fisiología de estos hongos. En la actualidad, más de 200 especies de hongos nematófagos han sido descritas y son un grupo con un alto potencial para ser usados como agentes de control biológico en la supresión de nematodos fitoparásitos (Qadri 1989, Qadry y Seleh 1990, De Leij y Kerry 1991, Khan y Akram 2000, Kerry 2000).

Los hongos nematófagos son habitantes del suelo que utilizan sus esporas o micelio para capturar nematodos vermiformes. Se les encuentra en diferentes tipos de sustratos y son capaces de sobrevivir en condiciones climáticas o nutricionales extremas en diferentes regiones geográficas del mundo (Gray et al. 1982). Su abundancia varía de 1,8-150 propágulos por gramo de suelo y esto obedece principalmente a la cantidad de materia orgánica, contenido de agua, tipo de suelo, temperatura y presencia de nematodos hospederos (Persmark et al. 1996).

De acuerdo a su estrategia de captura, se clasifican en hongos endoparásitos y depredadores (Cole y Kendrick, 1981). Los hongos endoparásitos se caracterizan por la formación de estructuras adhesivas o de ingestión (conidios adhesivos o ingeribles, o zoosporas móviles) que infectan nematodos o parasitan huevos formando un apresorio. Esta estructura es definida como un sistema hifal formada por el hongo que se adhiere a la cáscara del huevo segregando enzimas que permiten la penetración (Barrón 1977, López-Llorca y Robertson 1992, Morton et al. 2004).
Los hongos depredadores en cambio, se caracterizan por producir una red hifal extensa en el sitio donde se encuentren. Las estructuras predadoras de estos hongos pueden ser muy simples como los que forman hifas adhesivas septadas, redes adhesivas, ramificaciones adhesivas, botones adhesivos, anillos no constrictores o especializados que forman anillos que se encargan de sostener los nematodos vivos (Barrón 1977, Gray 1987).

La literatura menciona tres grupos más con alta capacidad supresora de nematodos. Uno de ellos, está relacionado con la especificidad de algunos hongos de atacar nematodos de acuerdo a su estado de madurez (Stirling 1991, Cave 1995, Obregón 2001, Obregón 2005), es decir, huevos, juveniles y hembras de nematodos principalmente de los géneros Heterodera, Globodera y Meloidogyne (Kerry y Jaffee 1997). Otro grupo incluye todos aquellos hongos que tienen la capacidad de producir toxinas (Jansson et al. 1997; Li et al. 2000). Por último, Luo et al. (2006) hallaron el hongo Stropharia rugosoannulata, que tiene la capacidad de matar nematodos dañando su cutícula mecánicamente con una estructura especial en forma de bola espinosa (acantocito).

Para asegurar un mayor éxito en el empleo de un agente de control biológico, es necesario conocer con más detalle su biología, descripción morfológica, detalles de su estructura y apariencia. Cada uno de estos detalles permitirá la descripción taxonómica de los antagonistas y contribuirá al acervo de conocimiento sobre dichos microorganismos y a un empleo más eficiente como biocontroladores (Orozco 2005).

En Costa Rica sin embargo, nunca se ha realizado un estudio enfocado a determinar mediante identificación taxonómica, los hongos nematófagos asociados al cultivo del arroz. Por lo anterior, el objetivo de la presente investigación fue identificar hongos nematófagos en fincas arroceras de Costa Rica.

\section{MATERIALES Y MÉTODOS}

La fase de aislamiento e identificación de hongos nematófagos se llevó a cabo en los Laboratorios de Nematología, Microorganismos de Suelo, Control Biológico y Fitopatología de la Escuela de Ciencias Agrarias de la Universidad Nacional, Heredia, Costa 
Rica. Se muestrearon fincas arroceras de secano en estado inicial, intermedio y final de cosecha en las regiones Huetar Atlántico, Pacífico Central y Chorotega, identificadas con problemas de nematodos. El estudio se realizó entre los meses de abril del 2008 a agosto de 2009 y se muestreó a los 0, 15, 45, 60 y 90 días del ciclo de cultivo. Cada sitio visitado, presentó condiciones climáticas, de altitud y propiedades químicas diferentes entre sí (Cuadro 1).

En total se realizaron trece giras de campo durante los meses de abril del 2008 a enero del 2009 (Figura 1). Para el aislamiento de los hongos nematófagos se delimitaron en cada finca tres áreas de muestreo de 4 $\mathrm{m}^{2}$ separadas entre sí por una distancia de $50 \mathrm{~m}$. En cada punto de muestreo, se recolectó con la ayuda de un barreno cinco submuestras a una profundidad de $20 \mathrm{~cm}$. Las muestras fueron almacenadas en bolsas de polietileno debidamente etiquetadas y trasladadas al Laboratorio de Ecología de Organismos del Suelo y Control Biológico para su debido procesamiento. Con la ayuda de un GPS se determinaron las coordenadas de cada punto de muestreo.

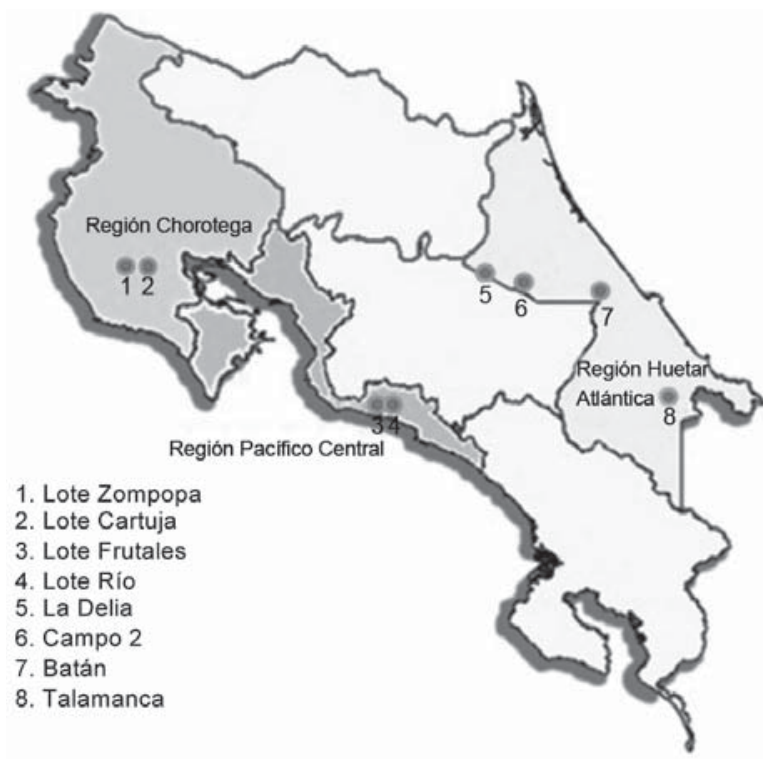

Figura 1. Sitios de muestreo de suelo para la identificación de hongos nematófagos de las regiones arroceras Chorotega, Pacífico Central y Huetar Atlántica. Costa Rica, 2008-2009.

Cuadro 1. Características ambientales y propiedades químicas de las tres regiones de Costa Rica, y los cantones donde están ubicadas las fincas arroceras muestreadas. Costa Rica. 2008-2009.

\begin{tabular}{|c|c|c|c|c|c|c|c|c|c|c|c|c|}
\hline \multirow[t]{2}{*}{ Región } & \multirow[t]{2}{*}{ Cantón } & \multirow[t]{2}{*}{ Distrito } & \multirow{2}{*}{$\begin{array}{c}\text { Precipi- } \\
\text { tación } \\
\text { (mm/ } \\
\text { anual) }\end{array}$} & \multirow[t]{2}{*}{$\begin{array}{l}\text { Altitud } \\
\text { (msnm) }\end{array}$} & \multirow{2}{*}{$\begin{array}{c}\text { Tempe- } \\
\text { ratura } \\
\left({ }^{\circ} \mathrm{C}\right)\end{array}$} & \multirow{2}{*}{$\begin{array}{c}\text { Hu- } \\
\text { medad } \\
\text { relativa } \\
(\%)\end{array}$} & \multirow{2}{*}{$\begin{array}{c}\text { Zona } \\
\text { de } \\
\text { vida }\end{array}$} & \multirow[t]{2}{*}{ Textura } & \multicolumn{2}{|c|}{$\begin{array}{c}\text { \% Materia } \\
\text { orgánica }\end{array}$} & \multicolumn{2}{|c|}{ pH $\mathrm{H}_{2} \mathrm{O}$} \\
\hline & & & & & & & & & Valor & Condición & Valor & Condición \\
\hline \multirow{4}{*}{$\begin{array}{c}\text { Huetar } \\
\text { Atlántico }\end{array}$} & $\begin{array}{c}\text { Pto. Viejo, } \\
\text { Sarapiquí }\end{array}$ & La Delia & \multirow{4}{*}{3525} & \multirow{4}{*}{50} & \multirow{4}{*}{27} & \multirow{4}{*}{84} & B.H.T. & $\begin{array}{l}\text { Franco } \\
\text { Arenoso }\end{array}$ & 8,9 & (Alto) & 5,8 & $\begin{array}{c}\text { Modera- } \\
\text { damente } \\
\text { ácido }\end{array}$ \\
\hline & Matina & Batán & & & & & & Arcilloso & 3,8 & (Medio) & 5,4 & Muy ácido \\
\hline & Cariari & Campo 2 & & & & & & Franco & 6,5 & (Alto) & 5,3 & $\begin{array}{l}\text { (Muy } \\
\text { ácido) }\end{array}$ \\
\hline & Talamanca & Shiroles & & & & & & Franco & 2,6 & (Medio) & 6,0 & $\begin{array}{l}\text { Ligeramen- } \\
\text { te ácido }\end{array}$ \\
\hline $\begin{array}{c}\text { Pacífico } \\
\text { Central }\end{array}$ & Parrita & Parrita & 3317 & 6 & 27 & 85 & $\begin{array}{c}\text { B.H.T. } \\
\quad *\end{array}$ & Arcilloso & 7,1 & (Alto) & 5,8 & $\begin{array}{c}\text { Modera- } \\
\text { damente } \\
\text { ácido } \\
\end{array}$ \\
\hline \multirow[t]{2}{*}{$\begin{array}{c}\text { Choro- } \\
\text { tega }\end{array}$} & Nicoya & Nicoya & \multirow[t]{2}{*}{1200} & \multirow[t]{2}{*}{20} & \multirow[t]{2}{*}{28} & \multirow[t]{2}{*}{70} & $\begin{array}{c}\text { B.H.S } \\
* *\end{array}$ & $\begin{array}{l}\text { Franco } \\
\text { Arenoso }\end{array}$ & 8,9 & (Alto) & 5,8 & $\begin{array}{l}\text { Modera- } \\
\text { damente } \\
\text { ácido }\end{array}$ \\
\hline & Cañas & Cañas & & & & & & Arcilloso & 3,8 & (Medio) & 5,4 & Muy ácido \\
\hline
\end{tabular}

\footnotetext{
*Bosque Húmedo Tropical (BHT), **Bosque Húmedo Seco (BHS).
} 


\section{Cultivo de nematodos bacteriófagos}

Nematodos bacteriófagos fueron utilizados como cebo para evidenciar la presencia de hongos nematófagos en las muestras de suelo. Se empleó la técnica de aislamiento y cultivo de nematodos bacteriófagos del orden Rhabditida utilizada por el Laboratorio de Nematología de la Universidad Nacional. El procedimiento consistió en colocar en una caja plástica una muestra de suelo y rebanadas de papa en la superficie. La caja con suelo se incubó durante una semana a una temperatura de entre 20 y $25^{\circ} \mathrm{C}$, este procedimiento permitió la colonización de las rodajas de papa por nematodos bacteriófagos presentes en el suelo. Posteriormente se procedió a la extracción e identificación preliminar de los nematodos y la transferencia a un medio de cultivo papa-dextrosa-agar (PDA diluido) a una concentración de $6,2 \%(2,44 \mathrm{~g} / \mathrm{l})$ para su reproducción en platos petri de $9 \mathrm{~cm}$. El procedimiento de transferencia a PDA diluido, se repitió transfiriendo cuatro discos de 1,0 cm de PDA con los nematodos deseados para la reproducción de una sola especie de nematodo. La complejidad taxonómica del orden Rhabditida no permitió identificar la especie.

\section{Aislamiento de hongos nematófagos}

Las muestras de suelo se procesaron mediante el método de "espolvoreado en placa" descrito por Barrón (1977) para el aislamiento de hongos nematófagos. La técnica consistió en utilizar por cada área de muestreo, cinco repeticiones colocando en cajas petri de $9 \mathrm{~cm}$ de diámetro con agar-agua (AA) y de 0,5 a 1,0 g de la muestra de suelo. Con la ayuda de una micropipeta se adicionó $1 \mathrm{ml}$ de una suspensión de entre 100-300 nematodos bacteriófagos previamente cultivados en medio PDA diluido. Las cajas se incubaron a temperatura ambiente $\left(23-26^{\circ} \mathrm{C}\right)$ y luz fluorescente. A partir del cuarto día de incubación las cajas se observaron diariamente usando un microscopio invertido, en busca de hifas, conidios, esporas o nematodos parasitados. Las observaciones se mantuvieron por una semana más con el fin de aumentar la posibilidad de encontrar alguna estructura fúngica. Una vez observada la presencia de hongos nematófagos, se procedió a su aislamiento y purificación. Con la ayuda de una asa micológica, se tomaron conidios, micelio o nematodos parasitados y se colocaron en medio PDA con tetraciclina $0,1 \%(\mathrm{p} / \mathrm{v})$ para evitar el crecimiento de bacterias. Las muestras se incubaron a temperatura ambiente y una semana después se verificó el crecimiento de los hongos. Posteriormente, las colonias obtenidas fueron transferidas a medio PDA sin antibiótico para su purificación y replicación. Los hongos identificados se conservaron en viales con PDA y en aceite mineral a $4^{\circ} \mathrm{C}$ para su posterior utilización en pruebas de patogenicidad (Kerry 2001).

\section{Identificación de hongos nematófagos}

La identificación taxonómica de los hongos nematófagos se realizó mediante la utilización de la clave "A key to the Nematode - Destroying Fungi" de Cooke y Godfrey (1964) y la clave de hongos imperfectos de Barnett y Hunter (1998). Se prepararon montajes semipermanentes en portaobjetos y una gota de colorante de contraste (lactofenol azul) con las estructuras fúngicas de cada uno de los hongos aislados. Las preparaciones se estudiaron en un microscopio compuesto a diferentes aumentos comparando su morfología con las descripciones disponibles. Se tomaron fotografías de las estructuras de reproducción.

\section{Textura, materia orgánica y pH del suelo}

Se determinó la textura (análisis mecánico del suelo) mediante el Método del Hidrómetro de Bouyoucos (1962), el contenido de Materia Orgánica se realizó mediante el método de Black (1965) y la determinación de acidez de suelo $(\mathrm{pH})$ mediante el potenciómetro con agua destilada.

\section{RESULTADOS Y DISCUSIÓN}

\section{Aislamiento e identificación de hongos nematófagos}

Un total de 52 aislamientos de hongos asociados al cultivo del arroz de tres regiones de Costa Rica, Región Pacífico Central (Parrita), Región Huetar Atlántica (Cariari, Sarapiquí, Batán) y Región Chorotega (Nicoya, Cañas) fueron documentados. De los hongos, 51 fueron identificados y uno fue clasificado como micelio estéril ya que no produjo estructuras de reproducción que facilitara su identificación. La Región Huetar Atlántica fue la que mostró el mayor número de hongos aislados 
(54\%), seguida de la Región Chorotega (32,5\%), y por último la Región Pacífico Central con 11,5\% (Cuadro 2).

Se aislaron 49 y tres especies, basado en sus descripciones microscópicas y aspectos morfológicos (Cuadro 3). El género más frecuentemente aislado e identificado fue Trichoderma sp. con 22\% (Figura 2A y 2B), seguido de Penicillium sp. 21\% (Figura 2C), Paecilomyces sp. 11\% (Figura 2D), Fusarium sp. 10\% (Figura 2E), F. oxysporum 6\%, Aspergillus sp. 6\% (Figura 2F), Monacrosporium sp. 4\% (Figuras 2G y 2H), Gonatobotrys sp. 4\%, Cladosporium sp., Phythium sp., Curvularia sp., Oomycete, Phoma sp., Geotrichum sp., micelio estéril, F. tabacinum con un $2 \%$. Algunos de estos géneros como Geotrichum, Phythium, Phoma, Gonatobotrys, Aspergillus, Curvularia y Penicilium son considerados patógenos de plantas sin acción deletérea sobre nematodos.
Cuadro 2. Número y porcentaje de cepas de hongos nematófagos obtenidos de suelos de fincas arroceras en las regiones Huetar Atlántica, Chorotega y Pacífico Central de Costa Rica. 2008-2009.

\begin{tabular}{lccc}
\hline Región & \multicolumn{3}{c}{ Colonias aisladas } \\
\cline { 2 - 4 } & $\begin{array}{c}\text { Identificadas } \\
(\%)\end{array}$ & $\begin{array}{c}\text { Desconocidas } \\
(\%)\end{array}$ & Total \\
\hline Huetar Atlántica & $28(54 \%)$ & - & 28 \\
Región Chorotega & $17(32.5 \%)$ & $1(2 \%)^{*}$ & 18 \\
Región Pacífico & $6(11.5 \%)$ & - & 6 \\
Central & & & \\
\hline Total & \multicolumn{2}{c}{52} & 52 \\
\hline
\end{tabular}

* Micelio estéril sin estructuras de reproducción.

Cuadro 3. Clasificación taxonómica de los principales hongos aislados de suelo de fincas arroceras y lugar de procedencia. Costa Rica. 2008-2009.

\begin{tabular}{|c|c|c|c|}
\hline Aislamiento & Altitud (msnm) & Coordenadas & Procedencia \\
\hline Trichoderma sp. & \multirow{2}{*}{30} & \multirow{2}{*}{$09^{\circ} 35^{\prime} 55,68^{\prime \prime} \mathrm{N} / 0,82^{\circ} 55^{\prime} 05,33^{\prime \prime} \mathrm{W}$} & \multirow{2}{*}{ Cañas. Guanacaste } \\
\hline Trichoderma sp. & & & \\
\hline Aspergillus sp. & 52 & $10^{\circ} 28^{\prime} 33,02^{\prime \prime} \mathrm{N} / 0,85^{\circ} 08^{\prime} 30,47^{\prime \prime} \mathrm{W}$ & Cañas. Guanacaste \\
\hline Fusarium oxysporum & 52 & $10^{\circ} 28^{\prime} 31,48^{\prime \prime} \mathrm{N} / 0,85^{\circ} 08^{\prime} 29,27^{\prime \prime} \mathrm{W}$ & Cañas, Guanacaste \\
\hline Phythium sp. & 198 & $10^{\circ} 05^{\prime} 38,9^{\prime \prime} \mathrm{N} / 0,85^{\circ} 28^{\prime} 53,9^{\prime \prime} \mathrm{W}$ & Nicoya, Guanacaste \\
\hline Micelio estéril & \multirow{2}{*}{194} & \multirow{2}{*}{$10^{\circ} 05^{\prime} 34,3^{\prime \prime} \mathrm{N} / 0,85^{\circ} 28^{\prime} 56,7^{\prime \prime} \mathrm{W}$} & \multirow{2}{*}{ Nicoya, Guanacaste } \\
\hline Penicillium sp. & & & \\
\hline Penicillium sp. & \multirow{2}{*}{184} & \multirow{2}{*}{$10^{\circ} 04^{\prime} 58^{\prime \prime} \mathrm{N} / 0,85^{\circ} 29^{\prime} 19,9^{\prime \prime} \mathrm{W}$} & \multirow{2}{*}{ Nicoya, Guanacaste } \\
\hline Trichoderma sp. & & & \\
\hline Geotrichum sp. & \multirow{2}{*}{179} & \multirow{2}{*}{$10^{\circ} 04^{\prime} 57,2^{\prime \prime} \mathrm{N} / 0,85^{\circ} 29^{\prime} 19,7^{\prime \prime} \mathrm{W}$} & \multirow{2}{*}{ Nicoya, Guanacaste } \\
\hline Penicillium sp. & & & \\
\hline Paecilomyces sp. & 173 & $10^{\circ} 04^{\prime} 56,7^{\prime \prime} \mathrm{N} / 0,85^{\circ} 29^{\prime} 19^{\prime \prime} \mathrm{W}$ & Nicoya, Guanacaste \\
\hline Paecilomyces sp. & 47 & $10^{\circ} 24^{\prime} 50,4^{\prime \prime} \mathrm{N} / 0,83^{\circ} 41^{\prime} 20,2^{\prime \prime} \mathrm{W}$ & Cariari, Guápiles \\
\hline Fusarium sp. & 47 & $10^{\circ} 24^{\prime} 50,6^{\prime \prime} \mathrm{N} / 0,83^{\circ} 41^{\prime} 22,5^{\prime \prime} \mathrm{W}$ & Cariari, Guápiles \\
\hline Penicillium sp. & 43 & $10^{\circ} 33^{\prime} 13,1^{\prime \prime} \mathrm{N} / 0,84^{\circ} 00^{\prime} 35,7^{\prime \prime} \mathrm{W}$ & Sarapiquí, Heredia \\
\hline Penicillium sp. & \multirow{8}{*}{43} & \multirow{8}{*}{$10^{\circ} 33^{\prime} 14,6^{\prime \prime} \mathrm{N} / 0,84^{\circ} 00^{\prime} 38,9^{\prime \prime} \mathrm{W}$} & \multirow{8}{*}{ Sarapiquí, Heredia } \\
\hline Monacrosporium sp. & & & \\
\hline Fusarium tabacinum & & & \\
\hline Paecilomyces sp. & & & \\
\hline Fusarium sp. & & & \\
\hline Fusarium sp. & & & \\
\hline Phoma sp. & & & \\
\hline Aspergillus sp. & & & \\
\hline
\end{tabular}


Continuación Cuadro $3 .$.

\begin{tabular}{|c|c|c|c|}
\hline Trichoderma sp. & 198 & $10^{\circ} 05^{\prime} 40^{\prime \prime} \mathrm{N} / 0,85^{\circ} 28^{\prime} 54^{\prime \prime} \mathrm{W}$ & Nicoya. Guanacaste \\
\hline Curvularia sp. & 5 & $09^{\circ} 51^{\prime} 63,2^{\prime \prime} \mathrm{N} / 0,84^{\circ} 32^{\prime} 79,7^{\prime \prime} \mathrm{W}$ & Parrita. Puntarenas \\
\hline Aspergillus sp. & 12 & $09^{\circ} 50^{\prime} 59,8^{\prime \prime} \mathrm{N} / 0,84^{\circ} 33^{\prime} 29,9^{\prime \prime} \mathrm{W}$ & Parrita. Puntarenas \\
\hline Gonatobotrys sp. & \multirow{3}{*}{12} & \multirow{3}{*}{$09^{\circ} 50^{\prime} 61,8^{\prime \prime} \mathrm{N} / 0,84^{\circ} 33^{\prime} 30,2^{\prime \prime} \mathrm{W}$} & \multirow{3}{*}{ Parrita. Puntarenas } \\
\hline Fusarium sp. & & & \\
\hline Fusarium oxysporum & & & \\
\hline Trichoderma sp. & \multirow{4}{*}{42} & \multirow{4}{*}{$10^{\circ} 01^{\prime} 47,3^{\prime \prime} \mathrm{N} / 0,83^{\circ} 20^{\prime} 24,2^{\prime \prime} \mathrm{W}$} & \multirow{4}{*}{ Batán. Limón } \\
\hline Paecilomyces sp. & & & \\
\hline Penicillium sp. & & & \\
\hline Monacrosporium sp. & & & \\
\hline Gonatobotrys sp. & \multirow{2}{*}{50} & \multirow{2}{*}{$10^{\circ} 24^{\prime} 50,6^{\prime \prime} \mathrm{N} / 0,83^{\circ} 41^{\prime} 22,5^{\prime \prime} \mathrm{W}$} & \multirow{2}{*}{ Cariari. Guápiles } \\
\hline Paecilomyces sp. & & & \\
\hline Trichoderma sp. & \multirow{2}{*}{40} & \multirow{2}{*}{$10^{\circ} 33^{\prime} 13,1^{\prime \prime} \mathrm{N} / 0,84^{\circ} 00^{\prime} 35,7^{\prime \prime} \mathrm{W}$} & \multirow{2}{*}{ Sarapiquí. Heredia } \\
\hline Penicillium sp. & & & \\
\hline Trichoderma sp. & \multirow{2}{*}{43} & \multirow{2}{*}{$10^{\circ} 33^{\prime} 13,8^{\prime \prime} \mathrm{N} / 0,84^{\circ} 00^{\prime} 36,8^{\prime \prime} \mathrm{W}$} & \multirow{2}{*}{ Sarapiquí. Heredia } \\
\hline Penicillium sp. & & & \\
\hline Paecilomyces sp. & \multirow{2}{*}{47} & \multirow{2}{*}{$10^{\circ} 33^{\prime} 14,6^{\prime \prime} \mathrm{N} / 0,84^{\circ} 00^{\prime} 38,9^{\prime \prime} \mathrm{W}$} & \multirow{2}{*}{ Sarapiquí. Heredia } \\
\hline Trichoderma sp. & & & \\
\hline Trichoderma sp. & \multirow{4}{*}{198} & \multirow{4}{*}{$10^{\circ} 05^{\prime} 40^{\prime \prime} \mathrm{N} / 0,85^{\circ} 28^{\prime} 54^{\prime \prime} \mathrm{W}$} & \multirow{4}{*}{ Nicoya. Guanacaste } \\
\hline Penicillium sp. & & & \\
\hline Oomycete & & & \\
\hline Fusarium oxysporum & & & \\
\hline Fusarium sp. & 12 & $09^{\circ} 51^{\prime} 63,2^{\prime \prime} \mathrm{N} / 0,84^{\circ} 32^{\prime} 79,7^{\prime \prime} \mathrm{W}$ & Parrita. Puntarenas \\
\hline Penicillium sp. & \multirow{6}{*}{254} & \multirow{6}{*}{$09^{\circ} 35^{\prime} 55,68^{\prime \prime} \mathrm{N} / 0,82^{\circ} 55^{\prime} 05,33^{\prime \prime} \mathrm{W}$} & \multirow{6}{*}{ Talamanca. Limón } \\
\hline Trichoderma sp. & & & \\
\hline Trichoderma sp. & & & \\
\hline Trichoderma sp. & & & \\
\hline Cladosporium sp. & & & \\
\hline Penicillium sp. & & & \\
\hline
\end{tabular}

\section{Aislamiento e identificación de hongos nematófagos}

Se comprobó la existencia de hongos nematófagos en las tres regiones arroceras muestreadas. Estos datos se suman a los obtenidos por Orozco (2005), en los cuales se encontró hongos nematófagos en ecosistemas de cuatro provincias del Valle Central y de la zona del Caribe de Costa Rica. En esta oportunidad se evidenció la presencia de Candelabrella musiformis, Arthobotrys oligospora y Dactylella sp. en sistemas agroforestales, pecuarios y bosques del país. Esta investigación confirma la ocurrencia de hongos nematófagos en zonas arroceras y su potencial como posibles agentes de control biológico de nematodos fitoparásitos.

$\mathrm{Al}$ analizar la diversidad de hongos nematófagos aislados, se determinó que Trichoderma sp. (23\%), Paecilomyces sp. (11\%), Fusarium oxysporum (6\%) y Monacrosporium sp. (4\%) se encontraron con mayor frecuencia. Trichoderma es el género de mayor aparición en las fincas arroceras, en particular presenta especies que pueden parasitar o ejercer efectos antagónicos sobre algunos hongos patógenos (Samaniego-Gaxiola y Chew-Madinaveitia 2007). Los cuatro géneros encontrados (Trichoderma sp., Paecilomyces sp., Fusarium sp. y Monacrosporium sp.) más $F$. 

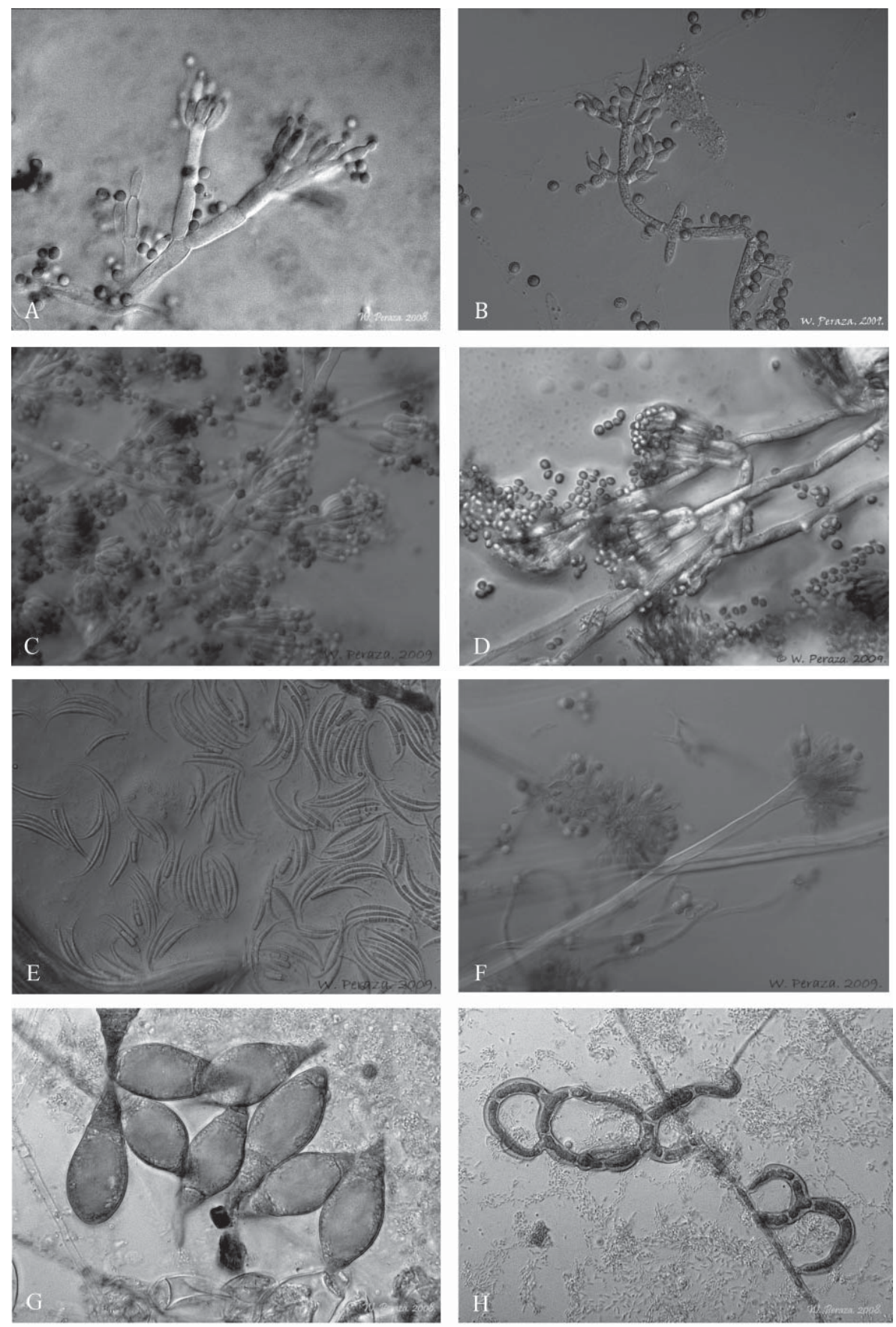

Figura 2. Fotomicrografías de hongos nematófagos aislados del suelo de zonas arroceras de Costa Rica, 2008-2009. A. Conidios y conidióforos de Trichoderma sp. 100x., B. Conidios y conidióforos de Trichoderma sp. 100x., C. Conidios y conidióforos de Penicillium sp. 100x., D. Conidios y Conidióforos de Paecilomyces sp. 100x., E. Macrocononidios de Fusarium sp. 100x., F. Conidio y conidióforo de Aspergillus sp. 100x., G. Conidios de Monacrosporium sp. 100x., H. Órgano de captura de Monacrosporium sp. 100x. 
oxysporum, han sido reportados en la literatura científica afectando poblaciones de nematodos parásitos de plantas. Sobre este mismo tema, Verdejo-Lucas, et al. (2002), encontraron Verticillium chlamydosporium, V. catenulatum, Fusarium spp., F. oxysporum, F. solani, Acremonium strictum, Gliocladium roseum, Cylindrocarpon spp., Engiodontium album y Dactylella oviparasitica asociados a masas de huevos de Meloidogyne spp. Adicionalmente, Núñez (2000) comprobó la presencia de tres géneros de Cladosporium sp. asociados a quistes de Globodera rostochiensis en plantaciones de papa (Solanum tuberosum) en la región del Cofre, Xalapa, Veracruz.

Otros géneros aislados como Fusarium sp., Cladosporium sp., Gonatobotrys sp., Penicilium sp., Asperillus sp., Geotrichum sp., y Curvularia sp. se consideran patógenos para plantas sin acción deletérea sobre nematodos. Moore (1996) coincide en afirmar que géneros como Fusarium sp., Chaetomium sp., Penicillium sp., y Alternaria sp., pueden afectar directamente algunos cultivos no así nematodos.

La técnica de espolvoreado en placa descrita por Barrón (1977) y utilizada en esta investigación, a pesar de ser cualitativa ya que no permite determinar la cantidad de propágulos presentes en el suelo, si reflejó la riqueza de hongos en las zonas estudiadas.

Las fincas muestreadas en su mayoría a excepción de una parcela orgánica en Talamanca, son representativas de una agricultura intensiva realizada en muchas zonas de Costa Rica. Estos sistemas productivos presentan suelos muy alterados debido al uso continuo de productos químicos y a la frecuencia con que se utilizan. Ambas circunstancias a pesar de que van en detrimento del equilibrio microbiano, quizás no intervinieron en la germinación de esporas ni tampoco inhibieron la actividad fúngica ya que se demostró la existencia de hongos en cada una de las regiones analizadas.

Una mayor aparición de estos antagonistas en la Región Atlántica pudo obedecer a que en esta parte del país las condiciones de precipitación y humedad son altas, por lo que quizás ha favorecido su actividad metabólica y por ende su aparición. Por otra parte, la alta precipitacion alrededor de los $3525 \mathrm{~mm} /$ anuales, temperatura cercana a los $27^{\circ} \mathrm{C}$ y una humedad relativa del $84 \%$ podría apoyar esta hipótesis. Además, Gray (1988) determinó que los hongos nematófagos son comunes en suelos agrícolas por lo que no es de extrañar la existencia de estos antagonistas en los sitios de muestreo y bajo estas condiciones. Rubner (1996), menciona que de estos hongos, las especies depredadoras crecen mejor a $30^{\circ} \mathrm{C}$ a diferencia de especies de las endoparásitas que lo hacen a temperaturas que rondan los $15^{\circ} \mathrm{C}$. Esta afirmación, explicaría la predominancia de hongos depredadores sobre endoparásitos en regiones tropicales como Costa Rica.

En este sentido, se optó por aislar hongos depredadores por ser más numerosos, diversos y fáciles de cultivar en masa y su capacidad reproductiva en medios de cultivo o en la naturaleza es comúnmente alta. Por otra parte, son eficientes y tienen gran versatilidad nutricional por lo que tienen la facilidad de sobrevivir en estado saprófito por largos periodos de tiempo. No obstante, son incapaces de formar trampas espontáneamente y son sensibles a efectos fungistáticos (Stirling 1991). Así mismo, a pesar de que muchos de los hongos endoparásitos son oportunistas obligados altamente específicos y se reproducen en el hospedero, presentan un rango de huéspedes más restringido.

Es importante mencionar que para el aislamiento, se utilizó únicamente la técnica de espolvoreado en placa descrita por Barrón (1977) exclusiva para hongos nematófagos depredadores y no para hongos endoparásitos de crecimiento lento. A pesar de ello, con esta técnica se determinó la existencia de una gran variedad y cantidad de hongos, sin embargo; para futuras investigaciones se podría emplear otras metodologías adicionales como la técnica de centrifugación diferencial o técnica del embudo de Baermann (Barron 1977) y así ampliar la diversidad de hongos nematófagos que podrían ser aislados.

\section{Textura, materia orgánica y pH}

Se determinaron valores de textura, $\mathrm{pH}$ y contenido de materia orgánica (M.O) en cada una de las fincas evaluadas. Con respecto a la textura, existió una predominancia de suelos francos en la Regiones Huetar Atlántico y Pacífico Central; y de suelos arcillosos en la Región Chorotega. El pH varió entre 5,3 a 6,0 y el contenido de materia orgánica fluctuó de 2,6 a 8,9 (Cuadro 1).

\section{Correlación entre textura, materia orgánica y pH en la aparición de hongos nematófagos}

Los hongos predominan en suelos ricos con alto contenido de restos vegetales donde pueden sintetizar y utilizar hidratos de carbono, alcoholes, 
ácidos orgánicos sencillos y hasta descomponer compuestos polimerizados, como la celulosa y la lignina; sin embargo, declinan rápidamente cuando las condiciones locales, especialmente de $\mathrm{pH}$ y de contenido de humedad cambian (Thompson y Troeh 1988). Adicionalmente, Julca-Otiniano et al. (2006) mencionan que los hongos toleran mejor situaciones ácidas y el escaso suministro de calcio que otros organismos en el suelo. Por otra parte, Wild (1992) menciona que gracias a su capacidad de adaptarse a condiciones desfavorables, los hongos pueden tolerar calor, sequía o mucha humedad. En esta investigación se confirmó que las fincas analizadas que mostraron una condición alta en el contenido de materia orgánica, Campos $2(8,9 \%)$, Nicoya $(7,1 \%)$ y La Delia $(6,5 \%)$ y con valores de $\mathrm{pH}$ en $\mathrm{H}_{2} \mathrm{O}$ de moderadamente ácido a muy ácido fue donde se aisló la mayor cantidad de hongos (60\% del total) (Cuadro 2). Adicionalmente, Hajieghrari et al. 2008) mencionan que el $\mathrm{pH}$ y la temperatura son parámetros claves para la manipulación en el crecimiento, esporulación y su capacidad saprofítica. Además, Medigan et al. (2000) afirman que los hongos tienden a tolerar ambientes más ácidos que las bacterias ya que la mayoría pueden crecer adecuadamente en un $\mathrm{pH}$ de 5,0 o inferior. Tanto la Región Huetar Atlántica como la Región Chorotega mostraron particularmente condiciones ambientales en muchos casos extremas de altas temperaturas, mucha lluvia, humedad y $\mathrm{pH}$ moderadamente ácido que concuerdan con la afirmación anterior.

Con respecto a los hongos y su relación con el contenido de materia orgánica en suelo, se ha determinado que algunos de ellos pueden sintetizar compuestos polifenólicos, contribuyendo a la formación de materia orgánica. Se determinaron valores de M.O que variaban entre 2,6 a 8,9 con condiciones de medio a alto, favoreciendo más esta última condición. Si bien la sucesión de material vegetal incorporado después de la cosecha en las fincas no ha sido un tema de estudio en Costa Rica, podría sugerir que la descomposición del rastrojo podría explicar la diversidad y riqueza de géneros de hongos que estarían interviniendo en el reciclado de nutrientes, procesos de respiración y descomposición de la materia orgánica. Así mismo, esta circunstancia podría contribuir a un cambio positivo de las condiciones físicas, químicas y abióticas del suelo y a la aparición de otros géneros en el suelo (Cooke 1962, Eren y Pramer 1978, Van den Boogert et al. 1994). Además, una mayor aireación mejora la estructura del suelo por lo que podría aumentar rápidamente las poblaciones edáficas, mejorar la capacidad amortiguadora de $\mathrm{pH}$ y la retención de agua y nutrientes.

De acuerdo a los muestreos realizados a los 0,15 , 45, 60 y 90 días se determinó una ocurrencia porcentual de hongos nematófagos del 25\% (0-30 días), 40\% (31-60 días) y un 35\% (61-90 días) respectivamente. Esta situación podría estar relacionada con el proceso de sucesión natural que se presentan en el ecosistema del suelo, probablemente acompañado de una disminución de la residualidad de los productos aplicados. Además la cantidad y calidad de exudados radicales procedentes de plantas maduras pudo tener un efecto positivo en la mayor diversidad microbiológica encontrada.

\section{AGRADECIMIENTOS}

El primer autor desea agradecer al proyecto de investigación "Programa Interinstitucional de Investigación en Biodiversidad y Ecología de Organismos de Suelo" financiado mediante fondos FES (Fondos de Educación Superior), así como a la Universidad Nacional. A Stephanie Orozco del Laboratorio de Fitopatología de la Escuela de Ciencias Agrarias de la Universidad por el proceso de identificación de los hongos.

\section{LITERATURA CITADA}

Barrón, GL. 1977. The nematode destroying fungi. Lancaster, Pennsylvania. Editorial Lancaster Press, Inc. $140 \mathrm{p}$.

Barnett, HL; Hunter, BB. 1998. Illustrated genera of imperfect fungi. Fourth Edition. 218 p.

Black, CA. 1965. Methods of soils analysis. Part II Chemical and microbiological properties. American Society of Agronomy, Inc. Publisher, Madison, Wisconsing USA. p. 902-904.

Bouyoucos, G. 1962. Hidrometer method improved for making particle size analysis of soils. Agronomy Journal. 54:464-465.

Cave, R. 1995. Manual para la enseñanza del control biológico en América Latina. Zamorano Honduras. Zamorano Academic Press. 188 p.

Cole, GT; Kendrick, B. 1981. Biology of Conidial Fungi. Vol. 2. Academia Press. EUA. 660 p. 
Cooke, RC. 1962. Behavior of nematode-trapping fungi during decomposition of organic matter in soil. Trans. Br. Mycol. Soc. 45:314-320.

Cooke, RC; Godfrey, BES. 1964. A key to the nematodedestroying fungi. Transactions in British Mycology Society. 47(1): 61-74.

Chen, S; Dickson, DW. 2004. Biological control of nematodes by fungal antagonist. In Chen, ZX; Chen, SY; D.W Dickson. eds. Nematology. Advances and perspectives, Volume II. Beijing, China. p. 979-1039.

De Leij, FAAM; Kerry, BR. 1991. The nematophagous fungus Verticillium chlamidisporium as a potential biological control agent for Meloidogyne arenaria. Revue Nematology 14:157-164.

Eren, J; Pramer, D. 1978. Growth and activity of the nematode-trapping fungus Arthrobotrys conoides in soil. In Loutit MW, Miles JAR. eds. Microbial ecology. Springer-Verlag, Berlin. p. 121-127

Gray, NF; Wyborn, CHE; Smith, RIL.1982. Nematophagous fungi from the maritime Antarctic. Oikos 38:194-201.

Gray, NF. 1987. Nematophagous fungi with special reference to their ecology. Biology Review 62:245-307.

Gray, N.F. 1988. Fungi attacking vermiform nematodes. In Pionar, Jr, G.O and Jansson, H.-B. eds. Deseases of Nematodes, vol II. CRC Press, Boca Raton. p. 3-38.

Hajieghrari, B; Torabi, M; Mohammadi, MR; Davari, M. 2008. Biological potential of some Iranian Trichoderma isolates in the control of soil borne plant pathogenic fungi. African Journal of Biotechnology 7:967-972.

Jansson, HB; Tunlid, A; Nordbring-Hertz, B. 1997. Biological control: nematode. In Anke T. ed. Fungal biotechnology. Chapman \& Hall, Weinheim. p. 38-50.

Jansson, HB; López-Llorca, LV. 2001. Biology of nematophagous fungi. In Misra JK. Horn BW eds. Trichomycetes and Other Fungal Groups. Enfield: 5 Science Publishers. p. 145-173.

Julca-Otiniano, A; Meneses-Florian, L; Blas-Sevillano, R; Bello-Amez, S. 2006. La materia orgánica, importancia y experiencias de uso en la agricultura. IDIESA (Chile) 24(1):49-61.

Kerry BA; Jaffee, BA. 1997. Fungi as biocontrol agents for plant parasitic nematodes. In Wicklow DT, Söderström B. eds. The Mycota, vol IV. Springer-Verlag, Berlin. p. 203-218.

Kerry, BR. 2000. Rhizosphere interactions and the exploitation of microbial agents for the biological control of plant parasitic nematodes. Annual Review Phytopathology 38:423-441.
Kerry, BR. 2001. Exploration of the nematophagous fungus Verticillium chlamydosporium Goddard for the biological control of root-knot nematodes (Meloidogyne spp.) In Fungi as Biocontrol Agents: Progress, Problems and Potential. CABI International, Wallingford, UK. p. 155-168.

Khan, MR; Akram, M. 2000. Effect of certain antagonistic fungi and rhizobacteria on wilt complex caused by Meloidogyne incognita and Fusarium oxysporum on tomato. Nematologia Mediterranea 28:139-144.

Li, TF; Zhang, KQ; Liu, XZ. 2000. Taxonomy of nematophagous fungi. Chinese Science and Technical Publishing. Science Press, Beijing. 18 p.

López-Llorca, LV; Robertson, WM. 1992. Immunocytochemical localization of a 32-kDa protease from the nematophagous fungus Verticillium suchlasporium in infected nematode eggs. Experimental Mycology 16:261-267.

Luo, H; Xuan, L; Guohong, L; Yanbo, P. Keqin, Z. 2006. Acanthocytes of Stropharia rusosoannulata. Function as a Nematode Attacking Device. Applied and Environmental Microbiology 72(4):2982-2987.

MAG (Ministerio de Agricultura y Ganadería). 2011. Política arrocera del gobierno de Costa Rica: Fortalecer la competitividad de pequeños y medianos arroceros (en línea). Ministerio de Agricultura y Ganadería, Ministerio de Economía Industria y Comercio, Ministerio de Comercio Exterior de Costa Rica. Consultado 15 nov 2010. Disponible en http://www.mag.go.cr/avisosynoticias/PPT_arroz_Version_11_01_2011.pdf.

Madigan, MT; Martinko, JM; Parker, J. 2000. Brock: Biología de los Microorganismos. Prentice Hall Iberia, Madrid, España. 1152 p.

Moore, L. 1996: Fundamentals of the fungi. Prentice Hall, Upper Saddle River, New Jersey. 4 ed. p. 433-438.

Morton, CO; Hirsch, PR; Kerry, BR. 2004. Infection of plant parasitic nematodes by nematophagous fungi: a review of the application of molecular biology to understand infection processes and to improve biological control. Nematology 62:161-170.

Núñez, AE. 2002. Aislamiento y evaluación de hongos nematófagos asociados a quistes de Globodera rostochiensis (Woll.) en la región del Cofre de Perote. Tesis Maestría. Universidad de Colima. México. 91 p.

Obregón, M. 2001. Manual de laboratorio de fitoprotección con el uso de productos naturales. INA. Cartago. $46 \mathrm{p}$.

Obregón, M. 2005. Seminario: Uso y manejo de biopesticidas en la agricultura. Oreamuno, Cartago. INA. Centro especializado en agricultura orgánica. 18 p. 
Orozco, M. 2005. Aislamiento y caracterización de hongos nematófagos como potenciales controladores biológicos de nematodos gastrointestinales para la producción animal. Tesis Maestría. Universidad Nacional. Heredia. Costa Rica. 104 p.

Persmark, L; Banck, A; Jansson, HB. 1996. Population dynamics of nematophagous fungi and nematodes in an arable soil: Vertical and seasonal fluctuations. Soil Biology and Biochemistry 28:1005-1014.

Qadri, AN. 1989. Fungi associated with sugar beet cyst nematode in Jerash Jordan. MSc Thesis, University of Jordan. 126 p.

Qadri, AN; Saleh, HM. 1990. Fungi associated with Heterodera schachtii (Nematoda) in Jordan. Nematologica 36:104-113.

Rubner, A. 1996. Revision of predacious hyphomycetes in the Dactylella-Monacrosporium complex. Studies in Mycology 39:1-134

Samaniego-Gaxiola, JA; Chew-Madinaveitia, Y. 2007. Diversidad de géneros de hongos del suelo en tres campos con diferente condición agrícola en la Laguna. Revista Mexicana de Biodiversidad 78(2):383-390.
Stirling, GR. 1991. Biological control of plant parasitic nematodes: Progress, problems and prospect. $\mathrm{CAB}$ International, Wallingford, UK. 282 p.

Thompson, LM; Troeh, FR. 1988. Los suelos y su fertilidad. Revert, S.A. Barcelona, España. p. 135-169.

Van Den Boogert, PH; Velvis, H; Hettema, CH; Bouwman, LA. 1994. The role of organic matter in population dynamics of the endoparasitic nematophagous fungus Drechmeria coniospora in microcosms. Nematologica 40:239-257.

Verdejo-Lucas, S; Ornat, C; Sorribas, FJ; Stchiegel, A. 2002. Species of root-knot nematodes and fungal egg parasites recovered from vegetables in Almería and Barcelona, Spain. Journal of Nematology 34(4):405408.

Wild, A. 1992. Condiciones de suelo y desarrollo de las plantas según Russell. Versión Española de P. Urbano Terrón y C. Rojo Fernández. Mundi-Prensa. Madrid, España. 1045 p.

Zopf, W. 1988. Zur Kenntnis der Infektions-Krankheiten niederer Thiere aud Pflanzen. Nova Acta Leopold Carol 52: 314-376. 